\title{
Transmission Performance of 433 Mhz Wireless Sensor Nodes in Through-the-Earth Communication
}

\author{
Xiaoqing $\mathrm{Yu}^{1, \mathrm{a}}$, Wenting $\mathrm{Han}^{1, \mathrm{~b}}$ and Zenglin Zhang ${ }^{1, \mathrm{c}}$ \\ Northwest A\&F University, Shaanxi,Yangling,712100, China \\ Ayuxiaoqing2006@163.com, 'bwentinghan200@126.com, 'zhangzenglin115@gmail.com
}

\begin{abstract}
Keywords: sensor nodes, transmission, signal strength, error rate, communication
Abstract. Wireless sensor nodes have the advantage of being low-cost easily deployed and of good mobility. However, the significantly high attenuation caused by soil is the main challenge for the communication of between the aboveground node and the underground node. In this paper, experimental measurements have been conducted with wireless sensor nodes at the frequency of 433 MHz. Experiments are run to examine the received signal strength of correctly received packets and the packet error rate for through-the-earth communication. The tests show the underground node burial depth and the volumetric water content of the soil are the most important factor to adversely affect the communication.
\end{abstract}

\section{Introduction}

Most existing monitoring detection systems located in information collection are connected by cable networks [1-3]. Drawbacks of these networks are complex cabling and difficulties associated with expanding them. Wireless sensor nodes have the advantage of being low-cost and easily deployed [4,5]. Deployment of low cost wireless sensor node is a useful technique for several applications ranging from early warning systems for natural disasters, ecosystem monitoring, real-time health monitoring, to homeland security and surveillance. When a signal propagates within a medium, it may be reflected, diffracted, and scattered. Each effect occurs to a different extent in various media, depending on factors such as wavelength and intensity of the wave, thickness and physical composition of the medium [6-10]. The ability to predict the signal strength provided by sensor nodes is not only useful to researchers but also a convenient capability in practice.

\section{Related Work}

In recent years, the research of through-the-earth communication technology come into a new active period with the development of communication technology. In the 1990s, Australia had developed emergency rescue command system, which was a real communication system used in practical application [11-13]. The author pointed out that three layers strati-graphic models could not reflect the local complex geological structure [14]. Barkand tested half duplex communication prototype system in a limestone mine and running coal mine, which showed that the system could satisfy the requirement of emergency communications [15]. In China, the through-the-earth communication system research is fewer, some test applications and theoretical research has been carried out. Mengtian Situ adopted pluralism FSK and decreased the information transmission rate to solve the weak signal reception problems [16]. Qingyi Zhang discussed the influence of system working frequency, medium conductivity, transmitting antenna size and placed in a large loop antenna case on underground electromagnetic wave propagation [17].

\section{Experiment Materials and Method}

In the wireless communication, the aboveground nodes send information management and requirements to the underground nodes, underground nodes need to communicate with the ground node to implement the data collection, management and relay, etc. Therefore, there are two different kinds of through-the-earth communication based on the transmitting node, the 
aboveground-underground communication communication and underground-aboveground communication. The diagram of through-the-earth communication structure is shown in Fig.1.

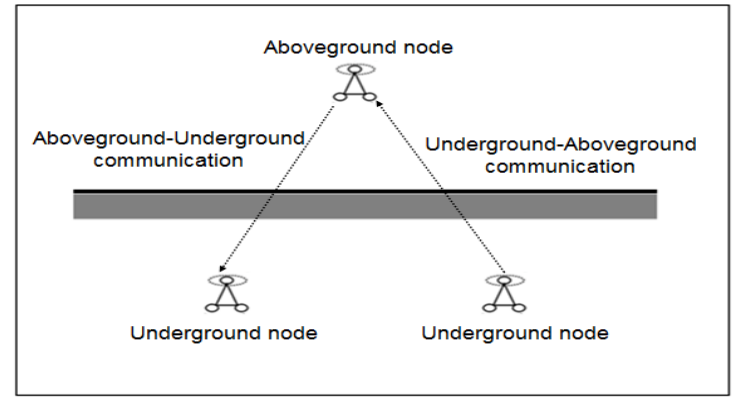

Fig.1 The diagram of through-the-earth communication structure

The through-the-earth communication experiments with 433MHz sensor nodes were carried out in the laboratory of the Research Institute of Water-saving Agriculture of Arid Regions of China in the Northwest Agriculture and Forestry University. The basic physical property index of the soil sample is shown in table 1.

Table 1. The basic physical property index of the soil sample

\begin{tabular}{cccc}
\hline \multirow{2}{*}{ Soil type } & \multicolumn{3}{c}{ Particle-sized fractions (\%) } \\
\cline { 2 - 4 } & Sand & Silt & Clay \\
Silty loam & 27.42 & 61.26 & 11.32 \\
\hline
\end{tabular}

In the trial, sensor nodes use 433MHz frequency. The output power were always set to maximum transmit power of $20 \mathrm{dBm}$. The antenna of sensors nodes are a standard one-quarter wavelength monopole antenna with $17 \mathrm{~cm}$ lengths, and the antennas are vertically oriented.

\section{Experiment results and discussion}

In the paper, the received signal strength and the packet error rate of electromagnetic wave transmission in the soil medium are studied under $433 \mathrm{MHz}$ frequency. The effects of node buried depth and volumetric water content of the soil parameters on received signal strength and packet error rate are established through Matlab in communication.

The Aboveground-underground Communication. The aboveground node is deployed on the aboveground $100 \mathrm{~cm}$ that is perpendicular to the underground node and horizontal distance $2000 \mathrm{~cm}$ with underground node. When the underground node burial depth and volumetric water content of the soil change at the same time, received signal strength changes in the range of $-53 \mathrm{dBm}$ to $-110 \mathrm{dBm}$, is shown in Fig.2.

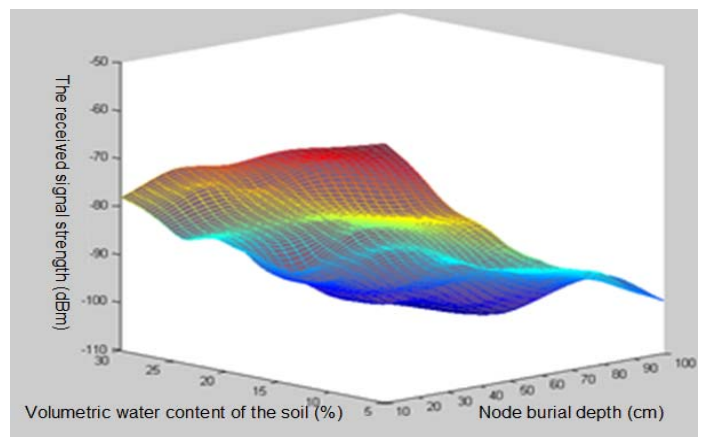

Fig.2. Effects of received signal strength in aboveground-underground communication

It can be included in Fig.2, that received signal strength changes with the change of underground node buried depth and volumetric water content of the soil. When soil moisture content is below 15\%, received signal strength is not lower than $-100 \mathrm{dBm}$ and communication quality is very good. The received signal strength reaches $-100 \mathrm{dBm}$ at different nodes buried depth $80 \mathrm{~cm}, 70 \mathrm{~cm}$ and $60 \mathrm{~cm}$ when soil moisture content gradually increase to the highest $30 \%$. The analysis is carried on through Matlab, relationship is established in Equation (1). 


$$
\begin{gathered}
R_{\mathrm{ss}}=-47.5967-0.7604 N_{d}-0.3330 S_{v}-0.0027 N_{d}{ }^{2}-0.0020 N_{d} S_{v}-0.0123 S_{v}{ }^{2} \\
R^{2}=0.9845
\end{gathered}
$$

where $R_{s s}$ is the received signal strength, $\mathrm{dBm} ; N_{d}$ is the underground node burial depth, cm; $S_{v}$ is volumetric water content of the soil, \%。 It can be seen from the equation (1), the changes of $N_{d}$ and $S_{v}$ have binary quadratic relationship on $R_{s s}$, goodness-of-fit $R^{2}$ is higher. When the aboveground node is deployed on the aboveground $100 \mathrm{~cm}$ that is perpendicular to the underground node and horizontal distance $2000 \mathrm{~cm}$ with underground node, the packet error rate is shown in Fig.3.

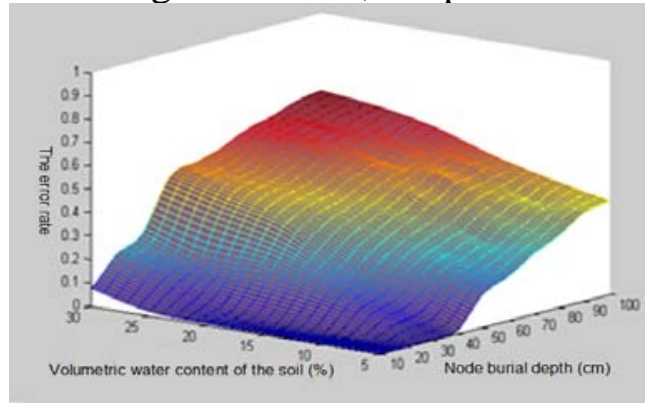

Fig.3. Effects of packet error rate in aboveground-underground communication

From Fig.3, the node buried depth and soil moisture content seriously affect transmission of electromagnetic wave in the soil medium, error rate has also changes and is not more than $70 \%$. When node buried depth increases from $10 \mathrm{~cm}$ to $100 \mathrm{~cm}$ and soil moisture content is below 20\%, the error rate within 50\%. The biggest error rate is less than $60 \%$ when soil moisture content changes. The analysis is carried on through Matlab, relationship is established in Equation (2).

$$
\begin{gathered}
E_{r}=-0.2591-0.0091 N_{d}-0.0101 S_{v}-0.0001 N_{d} S_{v}-0.0001 S_{v}{ }^{2} \\
R^{2}=0.9620
\end{gathered}
$$

where $E_{r}$ is the packet error rate. It can be seen from equation (2), the changes of $N_{d}$ and $S_{v}$ have binary quadratic relationship on $E_{r}$, goodness-of-fit $R^{2}$ is higher.

The Underground-aboveground Communication. Aboveground communication is one of the most important communications. The underground sensor node collects information and uploads to the aboveground node, the aboveground node analyzes and summaries the collected information. In the process of underground-aboveground communication test, wireless sensor node RF frequency is $433 \mathrm{MHz}$, the aboveground node is deployed on the aboveground $100 \mathrm{~cm}$ that is perpendicular to the underground node and horizontal distance $2000 \mathrm{~cm}$ with underground node, the change of the received signal strength and the packet error rate are analysed. The effect of underground node burial depth and volumetric water content of the soil on the received signal strength in the underground-aboveground communication is shown in Fig.4.

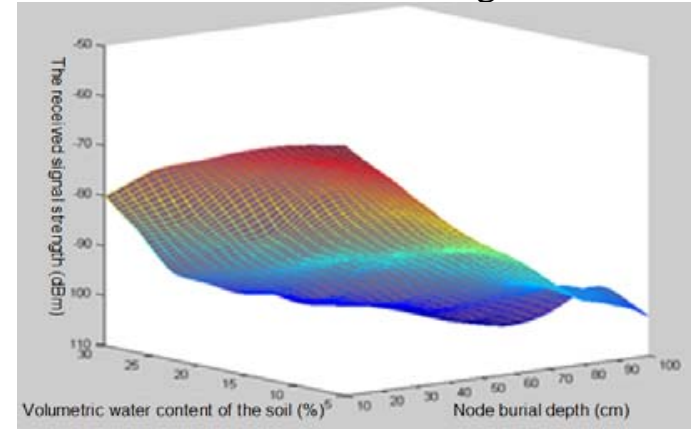

Fig.4. Effects of received signal strength in underground-aboveground communication

It can be observed in Fig.4, that the received signal strength is greater than $-100 \mathrm{dBm}$ when underground node burial depth changes from $10 \mathrm{~cm}$ to $100 \mathrm{~cm}$ and soil moisture content is below $15 \%$, and the received signal strength achieves the minimum $-110 \mathrm{dBm}$ when soil moisture content is more than $20 \%$. If the soil moisture content changes from $5 \%$ to $30 \%$, the received signal strength is greater than $-100 \mathrm{dBm}$ when underground node burial depth is less than $70 \mathrm{~cm}$ in underground-aboveground communication. 
Compared with the aboveground-underground communication, the received signal strength reduces the $2 \mathrm{dBm}$ to $4 \mathrm{dBm}$ in the underground-aboveground communication. The analysis is carried on through Matlab, relationship is established in Equation (3).

$$
\begin{gathered}
R_{s s}=-44.5594-0.8939 N_{d}-0.7176 S_{v}-0.0035 N_{d}^{2}-0.0058 N_{d} S_{v}-0.0101 S_{v}{ }^{2} \\
R^{2}=0.9819
\end{gathered}
$$

It can be seen from the equation (3), the changes of $N_{d}$ and $S_{v}$ have binary quadratic relationship on $R_{s s}$, goodness-of-fit $R^{2}$ is higher. When the aboveground node is deployed on the aboveground 100 $\mathrm{cm}$ that is perpendicular to the underground node and horizontal distance $2000 \mathrm{~cm}$ with underground node, Fig.5 reflects the change of error rate in the communication.

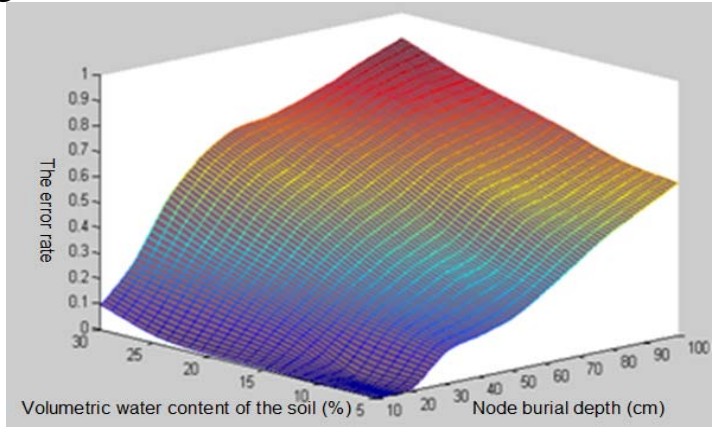

Fig.5. Effects of packet error rate in underground-aboveground communication

The Fig. 5 shows that error rate is less than $70 \%$ in the underground-aboveground communication when underground node burial depth changes from $10 \mathrm{~cm}$ to $100 \mathrm{~cm}$ and soil moisture content is below 20\%. when the soil moisture content gradually increases to the maximum $30 \%$, error rate reaches the maximum $90 \%$, the biggest error rate is less than $80 \%$.

Compared with the aboveground-underground communication, the error rate increases by about 2\%. The analysis is carried on through Matlab, relationship is established in Equation (4).

$$
\begin{gathered}
E_{r}=-0.2815-0.0241 N_{d}-0.0090 S_{v}-0.0001 S_{v}{ }^{2}+0.0001 N_{d} S_{v} \\
R^{2}=0.9769
\end{gathered}
$$

It can be seen from the equation (4), the changes of $N_{d}$ and $S_{v}$ have binary quadratic relationship on $E_{r}$, goodness-of-fit $R^{2}$ is higher.

\section{Summary}

Despite their potential, the proliferation of WUSN has been delayed by the unique challenges of the underground environment. Some of these challenges are related to the communication involving an underground node and an aboveground device. The through-the-earth communication is a promising new and the main technical means in the collection of underground information. In this work, we propose the application of through-the-earth communication. We have shown that the node burial depth and volumetric water content of the soil play an important role in the through-the-earth communication. Finally, the relationships are established through Matlab.

\section{Acknowledgements}

The research work was supported by China Postdoctoral Science Foundation funded project under Grant No. 2014M552495.

\section{References}

[1] M.K. Watfa, H. Al-Hassanieh and S. Salmen: A novel solution to the energy hole problem in sensor networks. Journal of network and computer applications. Vol. 36 (2013), pp. 949-958. 
[2] X.Q. Yu, W.H. Han and Z.L. Zhang: Overview of transmission characteristics in novel wireless underground sensor networks. International Journal of Future Generation Communication and Networking. Vol. 8 (2015), pp. 233-242.

[3] A.R. Silva and M.C. Vuran: Communication with above devices in wireless underground sensor networks: a empirical study. 2010 IEEE International Conference Proceedings. (2010), pp. 23-27.

[4] I.F. Akyildiz and E.P. Stuntebeck: Wireless underground sensor networks: Research challenges. Ad Hoc Networks. (2006), pp. 669-686.

[5] I.F. Akyildiz, M.C. Vuran and Z. Sun: Channel modeling for Wireless Underground Communication in Soil. Physical Communication. (2009)

[6] A. Buttafuoeo and E.B. Castrignan: Studying the spatial structure evolution of soil water content using multivariate geostatistics. Journal of Hydrology. Vol. 311 (2005), pp. 202-218.

[7] W.Q. Lee, W.K.G. Seah, H.P. Tan and Z. Yao: Wireless sensing without sensors: An experimental study of motion/intrusion detection using RF irregularity. (2010)

[8] D.G. Large, L Ball and A.J. Farstad: Radio transmission to and from underground coal mines-theory and measurement. IEEE Transactions on Communications. Vol. 21 (1973), pp. 194-202.

[9] R.G. Olsen and A.J. Farstad: Electromagnetic direction finding experiments for location of trapped miners. IEEE Tran. On Geoscience Electronics. Vol. 11 (1973).

[10] Z. Butler and D. Rus: Event-based Motion Control for Mobile Sensor Networks. IEEE Pervasive Computing. (2003).

[11]I.F. Akyildiz, W. Su, Y. Sankarasubramaniam and E. Cayirci: Wireless Sensor Networks: A Survey. Computer Networks. Vol. 38 (2002), pp. 393-422.

[12] A.K. Gogoi and R. Raghuram: Analysis of VLF loop antennas on the earth surface for underground mine communication. Antennas and Propagation Society International Symposium. (1996), pp. 962-965.

[13] J.J. Hao, H.L. Pang and H. Li: Experimental Studies on Transmission Characterization of Stratified Earth Channel in Through-the-Earth Communications with Elastic Wave. Advanced materials research. Vol. 734-737 (2013).

[14] N. Ayuso and J.A. Cuchi: Through-the-earth magnetic field propagation: modelling and experimental validation. Antennas and Propagation Society International Symposium 2006. (2006), pp. 680-683.

[15] T.D. Barkand, N.W. Damiano and W.A. Shumaker: Through-the-earth, Two-Way, Mine Emergency, Voice communication System. Industry Applications Conference. (2006), pp. 955-958.

[16] M.T. Situ: The technical scheme and key equipment for solving the difficult technical problems of underground radio communications. Engineering Science. Vol. 3 (2001), pp. 64-69.

[17] Q.Y. Zhang and J.M. Zhu: Study on propagation characteristics of the VLF through-the-earth communication channel. Chinese Journal of Radio Science. (1999), pp. 36-40. 